\title{
El compromiso como rasgo de personalidad y su relación con el burnout en docentes ecuatorianos
}

\section{The relationship between teaching commitment and burnout, an unresolved}

\author{
dilemma. Case study in Ecuadorian middle level teachers
}

Johana Espinel Guadalupe ${ }^{1}$, Erika Ruperti Lucero², Diana Aguilar Pita ${ }^{3}$

y Washington Miranda Vera ${ }^{4}$

${ }^{1}$ Psicóloga Industrial. Magíster en Educación. Doctoranda de la Universidad de Salamanca.

Profesora de la Universidad Estatal de Milagro, Ecuador. E-mail: jespinelg@unemi.edu.ec

${ }^{2}$ Psicóloga Educativa. Magíster en Psicología Educativa, mención Educación Especial.

Doctoranda de la Universidad de Almería. Profesora de la Universidad Estatal de Milagro,

Ecuador. E-mail: erupertil@unemi.edu.ec

${ }^{3}$ Psicóloga Educativa y Orientadora Vocacional. Máster en Orientación Educativa, Vocacional y Profesional. Doctoranda de la Universidad de Salamanca. Profesor de la Universidad Estatal de Milagro, Ecuador. E-mail: daguilarp@unemi.edu.ec

${ }^{4}$ Psicólogo Clínico. Magíster en Psicología Clínica. Profesor de la Universidad Estatal de Milagro, Ecuador. E-mail: wmirandav@unemi.edu.ec

Estudio realizado como parte de la programación del grupo de Investigación Análisis de Redes Sociales en Psicología e Investigación de la Universidad Estatal de Milagro.

$$
\text { Resolución N-007-Acta No04-DI-CI-2016. }
$$

Los autores agradecen a los docentes de la Unidad Educativa Velasco Ibarra de Milagro-Provincia del Guayas en Ecuador por el apoyo para realizar labores de investigación.

Unidad Educativa Velasco Ibarra de Milagro.

Provincia del Guayas, Ecuador.

\section{Resumen}

Los factores laborales pueden producir burnout en diferentes grados y a ciertos trabajadores; aunque todos estén expuestos al mismo contexto, no todos se afectan de igual manera, por lo que el objetivo de este estudio es determinar si el rasgo de personalidad-compromiso puede ser un elemento protector de burnout en el profesorado de nivel medio, considerando como hipótesis si sus niveles son independientes a los niveles de compromiso como rasgo de la personalidad. En este estudio participaron 134 docentes de la
Unidad Educativa Velasco Ibarra de la ciudad de Milagro, Ecuador, con edades entre 26 y 64 años. Luego de los permisos correspondientes, se les administró el Inventario de Preferencias y Personalidad PAPI $3 \mathrm{~N}$ de forma online, que contiene 12 necesidades y 14 roles descritos en 161 enunciados sobre el trabajador y su tarea, los cuales se ven enfocados en siete escalas, entre las que se encuentra el compromiso-escala que será utilizada para este estudio y que está conformada por la Necesidad de apoyar y el Rol de enfocarse al trabajo, y que forma parte del gran factor Responsabilidad. Para la variable burnout se utiliza el Cuestionario 
de Maslach para docentes (MBI-Ed), administrado de manera presencial, que consta de 22 ítems diseñados en escala de Likert y tres escalas: agotamiento emocional, despersonalización y realización personal. Se comprobó que los niveles de burnout dependen de los niveles del rasgo de personalidad compromiso $\left(\chi_{(4)}^{2}=11.919 ; p=.18\right)$ y los resultados podrían mostrar que se convierte en un elemento protector del burnout.

Palabras clave: compromiso, burnout, personalidad laboral, rasgo, despersonalización.

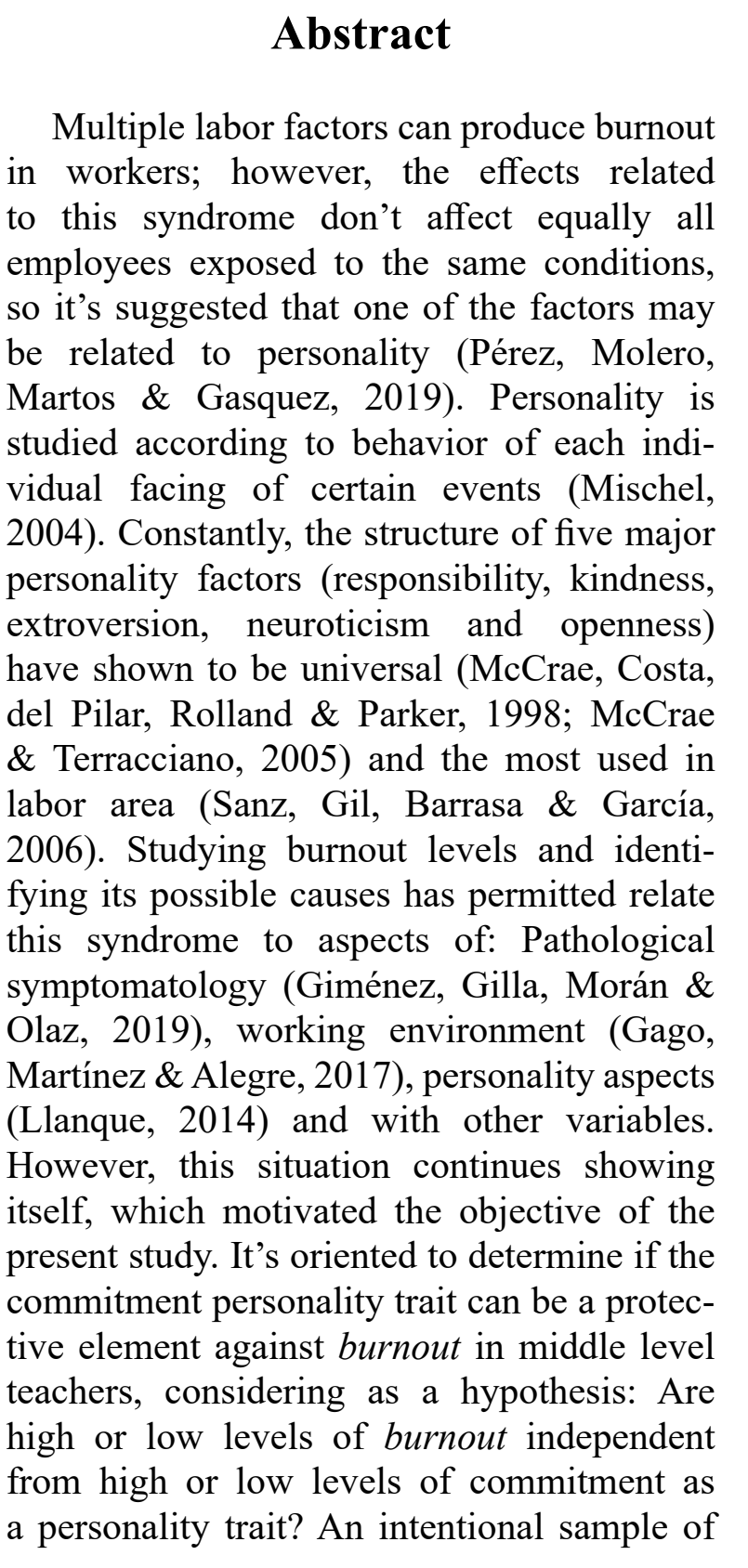

134 middle level teachers was used, between the ages of 26 and 64, in which $64 \%$ were women and the rest were men. To those who it was administered, after the corresponding permissions, The PAPI 3N Preferences and Personality Inventory online, which contains 12 needs and 14 roles described in 161 statements about the workers and their task, focus on seven scales. One of the great factors of the personality is responsibility, which forms the scale of the Commitment, which will be used for this study and that is conformed by the need to support and the role to focus on work, these personality indicators are those that, according to the authors, are associated to the teachers' activity. For the burnout variable, is used the Maslach Questionnaire for Teachers (MBI-Ed), which is administered in a face-to-face way, and consists of 22 items designed in a Likert scale and 3 scales; emotional exhaustion, depersonalization and personal accomplishment. Among the most important results we found a relationship between the commitment personality trait and the burnout levels existing in people (-.315) as well as with emotional exhaustion (-.195) and depersonalization (-.202), coinciding with the contribution of Sánchez (2013) and with Pérez, Molero, Martos and Gásquez (2019), who mention a causal relationship between both, the hypothesis test allowed the acceptance of the alternative hypothesis $\left(\chi_{(4)}^{2}=\right.$ $11.919 ; p=.018)$. It can be concluded that the compromise personality trait becomes a protective element against burnout.

Keywords: commitment, burnout, work personality, trait, despersonalization.

\section{Introducción}

Los diversos factores laborales pueden producir burnout en los trabajadores, sin embargo, los efectos relacionados con este síndrome no afectan por igual a todos los colaboradores expuestos a las mismas condiciones, por lo que se plantea que una de las razones para que esta dinámica sea distinta 
en un mismo sector de trabajo puede estar relacionado a la personalidad (Pérez, Molero, Martos y Gásquez, 2019).

La palabra "personalidad" proviene del latín persona y hace referencia a la máscara que se utiliza en el teatro para representar diversos papeles (Lewis y Anderson, 1998). Para estudiarla es necesario medirla de acuerdo al comportamiento del individuo ante ciertos sucesos (Mischel, 2004). De forma constante, la estructura de cinco grandes factores ha demostrado ser universal (McCrae, Costa, del Pilar, Rolland y Parker, 1998; McCrae y Terracciano, 2005) y la más utilizada en el área laboral (Sanz, Gil, Barrasa y García, 2006).

Los cinco factores que conforman la teoría del Big Five son: Apertura a la experiencia, Responsabilidad, Extraversión, Amabilidad y Neuroticismo; además, dependiendo del instrumento utilizado tendrá a la vez varias escalas. Dentro del factor Responsabilidad existen otros subfactores, entre ellos el compromiso, conformado por la necesidad de apoyar y el enfoque al trabajo (Cubiks, 2014).

El gran factor, responsabilidad, se refiere a la persistencia, el control y la motivación en tareas dirigidas a alcanzar un objetivo (Navarro Abal, 2009) y, bajo este criterio, el compromiso organizacional tiene dos alternativas: en primera instancia cuando el empleado está comprometido con la organización y busca la resolución de los problemas que aumentan sus niveles de ansiedad y, en segundo lugar, cuando el compromiso organizacional se convierte en técnicas de afrontamiento de situaciones estresantes (Glazer y Kruse, 2008).

Algunos estudios mencionan que el rasgo del compromiso, que se deriva del gran factor responsabilidad, también es susceptible al paso del tiempo, con una correlación entre mayor edad y mayor compromiso. Este es considerado como uno de los factores al deterioro del contrato psicológico con el paso de los años (Böhrt, Solares y Romero, 2014). Asimismo, los sistemas de turno y la antigüedad laboral se relacionan con el compromiso organizacional (Castro, Martínez, Robledo y Sierra, 2014) de tal manera que las virtudes organizacionales pueden influir en él (Lupano y Castro, 2018).

También se encontraron estudios como el de Maldonado, Ramírez, García y Chaires (2014) en los que, de manera general, muestran resultados de un compromiso medio de los profesores con su organización, pero no encuentran relación entre el compromiso y otras variables como género, estado civil, edad e incluso antigüedad o aquellas que vinculan al compromiso laboral con el desgaste físico y psicológico (Ruiz, Pando, Aranda y Almeida, 2014).

Estudiar los niveles de burnout y buscar sus posibles causas ha permitido relacionar este síndrome con aspectos de: sintomatología patológica (Giménez, Gilla, Morán y Olaz, 2019), clima laboral (Gago, Martínez y Alegre, 2017), aspectos de personalidad (Llanque, 2014) y con otras variables. Sin embargo, esta situación sigue presentándose, por lo que se consideró, como objetivo del presente estudio, determinar si el rasgo de personalidad-compromiso, se puede convertir en un elemento protector de niveles altos de burnout en el profesorado de nivel medio.

El burnout en la actualidad pertenece a uno de los malestares psicoemocionales derivados por factores estresantes, que da origen al surgimiento de otros problemas en el mundo laboral (Marenco-Escuderos y Ávila-Toscano, 2016; Prada-Ospina, 2019). A pesar de que ha sido estudiado, todavía son muy pocos los estudios que reflejan aspectos psicológicos y fundamentos fisiológicos del burnout (Heinemann y Heinemann, 2017).

Marsollier (2013) manifiesta que a esta sensación de ver proyectada sus ansiedades fuera de sí se le suele llamar "despersonalización”, es decir prescindir o dividir parte de sí mismo para poder soportar un poco más la realidad, de manera que el individuo se siente separado o disociado, lo cual consiste en diferencias en las experiencias sensoriales, adormecimiento emocional, distor- 
sión temporal, mnémica o incapacidad de evocación, disociación que suele darse entre uno mismo y el propio cuerpo o entre uno mismo y sus emociones. En cuanto a situaciones estresantes, la guía diagnóstica CIE 10 (World Health Organization, 2000) refiere que pueden expresarse con un malestar mayor a lo esperado llevar al deterioro significativo de la actividad social o laboral, asociando directamente a la sintomatología con el factor estresante y no a otro trastorno o a una situación de duelo. En dicho apartado menciona también que, de suspenderse el motivo de estrés, "los síntomas no persisten más allá de seis meses". Asimismo, se puede encontrar semejanza clínica en el llamado Trastorno de ansiedad generalizada (World Health Organization, 2000), debido a que, según los criterios, existe ansiedad y preocupación excesiva, además de expectación aprehensiva, sobre una amplia gama de acontecimientos o actividades (como el rendimiento laboral o escolar), que se prolongan más de seis meses, periodo en el cual, al individuo le es difícil controlar este estado de constante preocupación. Las respuestas físicas a este trastorno están relacionadas con la fatigabilidad, irritabilidad, tensión muscular y alteraciones del sueño.

Los docentes con burnout, causado por un estrés prolongado e insidioso, por condiciones individuales y de la organización, no facilitan el desempeño académico del estudiante (Hederich y Caballero, 2016), situación que coincide con la categoría $4 .^{\circ}$ y considerada en el Análisis Multiaxial según el Manual de Diagnóstico Estadístico (DSM). Esta categoría menciona que los problemas laborales y aquellos relativos a la enseñanza y las dificultades en la actividad social, laboral o escolar (Association American Psychiatric, 1995) producen desgaste profesional que consiste en un proceso de pérdida lenta, pero considerable, del compromiso y la implicación personal en la tarea que se realiza, situación que puede existir y actuar en todas las facetas de la persona.

Los efectos del burnout repercuten en los estados de salud de quienes lo padecen, así como lo demuestra un estudio realizado en 2009 en Chile, en el que se evidenció que al menos un tercio de los docentes de Santiago presentaban niveles altos en la escala de burnout. La consecuencia de aquello se puede encontrar en las respuestas psicológicas de los docentes, y puede afectar el clima de cordialidad dentro del aula y en el porcentaje de enfermedades presentadas por los profesores estudiados que superan la media nacional (Cornejo Chávez, 2009).

Otro estudio manifiesta que el síndrome de burnout suele repercutir en aquellas profesiones en las que se genera más contacto social o que tienen funciones de carácter de asistencia. Por tal razón, el profesorado padece de un agotamiento emocional que origina una despersonalización (Martínez Ramón, 2015), considerando que el ser humano, como entidad biopsicosocial, necesita apoyo social, para evitar los síntomas del burnout (Moreno, González y Garrosa, 2001).

La presencia del burnout en docentes universitarios también ha sido demostrada: en un estudio mexicano se observó el $63.5 \%$ de prevalencia del síndrome de burnout en docentes que, además, presentaron un agotamiento emocional del $47.4 \%$; despersonalización del $26.9 \%$ y el $76.9 \%$ presentó niveles bajos de realización personal en el trabajo (Ruiz de Chávez Ramírez, Pando Moreno, Aranda Beltrán y Almeida Perales, 2014). De la misma manera, en el estudio realizado por Arquero, Donoso, Hassall y Joyce (2006) se evidenció que un $28 \%$ mostraba niveles altos de burnout.

Por otra parte, se ha encontrado relación entre el burnout, la antigüedad en el puesto y los estados ansiosos, por lo que se predice que en las personas con más antigüedad y con niveles bajos de realización personal se encontrarán altos índices de ansiedad (Sagripanti, Gonzalez, Messi, Romero y Khlie, 2012). También se vincula el burnout con otros factores, como las variables sociodemográficas y laborales (Giménez, Gilla, Morán y Olaz, 2019). 
El síndrome de burnout se presenta en todo tipo de trabajo. Es así que, al estudiar a un grupo de 58 profesionales encargados de la seguridad y salud ocupacional, se encontraron resultados que indican que el $87 \%$ de la muestra presentó índices moderados de este síndrome, con el factor de baja realización personal como el que tuvo mayor incidencia (Arias Gallegos y Núñez Cohello, 2015).

Lo mismo sucedió en un estudio realizado a personal sanitario, en el que, de una muestra de 300 personas, el $27 \%$ obtuvo una baja realización personal, asociada a factores psicosociales como una alta exigencia laboral (42\%) y remuneración baja (43.7\%) (Silva Henao, Gutiérrez Strauss, Pando Moreno y Tuesca Molina, 2014). Esto se confirma en el estudio de Aldrete, Navarro, González, Contreras y Pérez (2015), que suma a estos factores psicosociales el tipo de tarea y la carga de trabajo (Pando, Gascón, Varillas y Aranda, 2019), motivo por el cual se considera al burnout como uno de los daños de tipo laboral y psicosocial más importantes en la actualidad (Spontón, Trógolo, Castellano y Medrano, 2019).

La literatura sobre este tema ha mostrado cómo el colectivo de profesorado tiene niveles bajos o muy bajos de realización personal, que es uno de los factores del burnout (Cornejo, 2009; Ruiz, Pando, Aranda y Almeida, 2014), a lo que Arquero, Donoso, Hassall y Joyce (2006) agregan que este denominador común se ve reflejado en diferentes contextos y culturas. Así también, en un estudio realizado en el área de la salud, las enfermeras con baja despersonalización, que es otra dimensión de burnout, utilizan en mayor medida el análisis lógico, y se asume que se lo hace como una estrategia que permite afrontar situaciones estresantes (Popp, 2008).

Rodríguez y De Rivas (2011) refieren que la acumulación del estrés puede llevar a su cronicidad, y tiende a desbordarse cuando el sujeto agota sus defensas y percibe una situación de desgaste, que influye multidimensionalmente al sujeto en toda su esfera biopsico- social. Asimismo, señalan que las diferencias individuales y la aparición de los estresores varían según la individualidad del sujeto tanto en sus respuestas como en sus defensas.

En cuanto a la personalidad y su vinculación con el burnout, se han encontrado relaciones entre factores afectivos y de personalidad con el bienestar laboral del profesorado, lo cual indica que determinados elementos sociales y de personalidad, como la amabilidad, actúan como un predictor de la realización personal y la satisfacción laboral (Laca, Mejía, Yáñez y Mayoral, 2011). Al analizar los cinco grandes factores de personalidad, se encontró que el neuroticismo está muy relacionado con el agotamiento en el trabajo y que este gran factor sumado a la responsabilidad se vincula con el compromiso laboral (Jeong Kim, Hyun Shin y Swanger, 2009).

Otros estudios reflejan cómo el neuroticismo, relacionado con las emociones, tiene vinculación con niveles altos de agotamiento (Narumoto y otros, 2008). Estos resultados fueron confirmados por Ortega y Ortiz (2014), quienes también conectan este gran factor de personalidad con dimensiones del burnout como el agotamiento y la realización personal (Kokkinos, 2007). Otras investigaciones relacionan el factor de personalidad liderazgo con la realización personal vinculada con el burnout (Contreras, Espinal, Pachón y González, 2013), motivo por el cual se ha considerado a la personalidad como un moderador de los niveles de burnout (Ortega y Ortiz, 2014).

Morán Astorga (2005) refiere en su estudio que el neuroticismo es la dimensión de la emocionalidad o la tendencia general a experimentar emociones negativas, siendo el polo contrario la estabilidad emocional, el mismo que puede influir en astenia, estado de agotamiento general. Además, refiere que las personas con mayor desequilibrio emocional (alto neuroticismo), son las más propensas a quemarse en el trabajo (Morán Astorga, 2005).

García et al. (2009) refieren en su estudio que, del análisis correlacional, el agotamiento 
emocional se asocia positivamente con el neuroticismo y negativamente con los otros cuatro factores del Big Five y sus resultados confirman la idea de que las personas que puntúan alto en neuroticismo. Esto significa que son más inestables emocionalmente, son más vulnerables al estrés psicosocial y es más probable que respondan con agotamiento emocional (García Izquierdo, Ramos Villagrasa y García Izquierdo, 2009).

Los riesgos psicosociales que se dan en el trabajo están relacionados con el compromiso, tal como lo demuestran estudios realizados en una organización del sector de la salud, cuyos resultados permiten deducir que existe un estado mental positivo relacionado con el compromiso y el trabajo en general (Arenas y Andrade, 2013).

De igual manera, al estudiar la relación entre el compromiso y el burnout en el personal de un hospital, se encuentra un impacto positivo entre ambos de un .02 (Gholipour, Reza, Anvari y Kalali, 2011). En otro estudio, en el que la muestra refleja niveles óptimos de compromiso (niveles medios, alto y muy alto), se observa que tienen su origen en la cultura de la organización, en la que se resalta el rasgo compromiso como uno de sus valores institucionales (Suárez y Mendoza, 2014).

Ciertas dimensiones de la personalidad tienen más influencia en el bienestar subjetivo de las personas que algunas condiciones de vida y del entorno (Mejía, Laca y Gondra, 2009). Simkim y Pérez (2018) indican que las personas con mayor neuroticismo tienden a carecer de confianza en su capacidad para llevar a cabo eficazmente las tareas requeridas y encuentran dificultades para la resolución de problemas, lo que afecta directamente su autoeficacia (Simkin y Pérez Marín, 2018).

Vallejo y Pérez (2018) mencionaron que, en cuanto al estudio de la personalidad, muchos autores encuentran que dentro del modelo de los Cinco Grandes, el neuroticismo es el rasgo de personalidad más consistentemente asociado con psicopatologías. En un estudio para analizar las diferencias entre el neuroticismo, la comorbilidad y el afrontamiento en un grupo de pacientes diagnosticados con trastorno adaptativo, se comprobó que aquellos con evolución desfavorable presentaban mayores niveles de neuroticismo, más presencia de rasgos comórbidos de personalidad patológica y un afrontamiento más desadaptativo (Vallejo Sánchez y Pérez García, 2018).

Al relacionar la inteligencia cultural, el compromiso organizacional y el burnout se encuentran correlaciones positivas aproximadas a .455 , que demuestran que al aumentar el compromiso organizacional se disminuye el burnout y, por lo tanto, la empresa será más eficiente (Dolatshah y Hosseini, 2016). Esto confirma los resultados obtenidos por Gordon (2004), quien manifestaba que la realización personal será mayor si el compromiso organizacional es alto. Sin embargo, Garrosa, Rainho, Moreno y Monteiro (2010) no encontraron una relación entre el compromiso y el burnout.

La hipótesis planteada sugiere que los niveles de burnout dependen de los niveles del rasgo de personalidad compromiso que, a la vez, forma parte del gran factor de personalidad responsabilidad, constituido por la necesidad de apoyar a otros y por el rol de enfocarse al trabajo. Esto mantiene la línea de lo planteado por Pérez, Molero, Martos y Gásquez (2019) sobre la existencia de una relación negativa entre la dimensión de burnout denominada "agotamiento emocional" y cuatro de los cinco grandes factores de la personalidad, entre ellos la responsabilidad o también conocida como conciencia. Por ello, el objetivo de este estudio es determinar si el rasgo de personalidad compromiso puede ser un elemento protector de burnout en el profesorado de nivel medio, considerando como hipótesis si los niveles de burnout son independientes a los niveles de compromiso como rasgo de la personalidad. 


\section{Método}

\section{Participantes}

El estudio es de tipo cuantitativo de corte transversal con análisis multivariante con el propósito de medir, explicar y predecir la relación entre las variables. Se utilizó una muestra intencional, para lo que se eligió un centro educativo público, representantivo a nivel de la zona, con bachillerato internacional, reconocido como referente por brindar excelentes profesionales a la sociedad. La muestra la conformaron 169 participantes, pero solo se incluyeron 134 docentes que fueron quienes llenaron todos los instrumentos de medición. Con edades que fluctúan entre 26 y 64 años, la distribución de la muestra por sexo es de 64 $\%$ mujeres y $36 \%$ varones, con un porcentaje significativo de docentes que tienen 14 años de antigüedad dedicados a la docencia.

\section{Instrumentos}

El primer instrumento utilizado fue el Inventario de Preferencias y Personalidad PAPI 3N (Cubiks, 2014) validado al castellano por Sanz, Gil, Barrasa y García (2006), cuya fiabilidad por escala es superior $(\alpha=.70)$ $y$ en nuestro estudio un $(\alpha=.94)$ en escala general. Ellas contienen 12 necesidades y 14 roles descritos en 161 enunciados sobre el trabajador y su tarea, los cuales se ven enfocados en siete escalas; (1) Influencia y Ambición; (2) Organización y Estructura; (3) Ideas y Cambio; (4) Interacción; (5) Dinamismo; (6) Compostura, y (7) Compromiso, escala que será la utilizada para este estudio. Esta escala se enfoca en los deseos del evaluado para apoyar la gestión de la organización para la cual trabaja, así como en el nivel de compromiso y motivación para realizar el trabajo y que se subdivide, a la vez, por la necesidad de apoyar y el rol de enfocarse al trabajo.

Se evaluaron los niveles de burnout por medio del Cuestionario de Burnout de Maslach para docentes (MBI-Ed) que consta de tres dimensiones que son: (1) agotamiento emocional (AE), con nueve ítems; (2) despersonalización (DP) con cinco ítems, y (3) realización personal (RP) con ocho ítems. Los 22 ítems están diseñados en escala de Likert de siete puntos: 1 (nunca), 2 (algunas veces al año o menos), 3 (una vez al mes o menos), 4 (algunas veces al mes), 5 (una vez a la semana), 6 (varias veces a la semana), 7 (todos los días) (Maslach y Jackson, 1986).

Para comprobar la fiabilidad de los resultados se aplicó la prueba de análisis alfa de Cronbach, en cada una de las dimensiones: (1) en la dimensión agotamiento emocional $(\alpha$ $=.720)$; (2) en la dimensión de despersonalización, compuesta por cinco ítems, se obtuvo un índice de fiabilidad de $\alpha=.686$; (3) en la dimensión de realización personal un $\alpha=$ .741 ; y (4) en escala general un $\alpha=.719$.

\section{Procedimiento}

Para la aplicación de los instrumentos de medición, se solicitó el permiso correspondiente a las autoridades del centro educativo y se informó a los participantes sobre el objetivo del estudio. La aplicación del MBI-Ed fue realizada de manera presencial y se convocó a los docentes a una sala dentro de la institución para que puedan llenar el instrumento de medición. Para la aplicación del test PAPI, cuya aplicación es virtual, se solicitaron los correos electrónicos de los docentes al Departamento de Talento Humano y se envió por esa vía la información sobre la prueba. Una vez que los docentes conocieron la información de la prueba, se procedió a ingresar a la plataforma de la empresa Cubiks para enviar vía correo electrónico la invitación con el respectivo link para su realización. Con los datos obtenidos a través de la plataforma de Cubiks, se procedió a incluir en la muestra a los participantes que completaron la prueba. El análisis de los datos se realizó utilizando Excel 2010 y SPSS Statistics v25, desde el cual se hicieron análisis descriptivos, de correlación de Pearson y análisis de regresión. Se 
usó la prueba de comprobación chi cuadrado comparando la $\chi_{\text {prueba }}^{2} \operatorname{con} \chi_{\text {tabla }}^{2}$ para aprobar o descartar la hipótesis planteada.

\section{Análisis de los resultados}

Los datos de cada uno de los instrumentos fueron ingresados al programa estadístico SPSS v.25, se consideraron los puntajes totales por cada una de las pruebas de medición de variables $\mathrm{y}$, una vez obtenido los puntajes totales, se realizaron los cortes de las medidas de posición de acuerdo a lo establecido por los autores de cada uno de los instrumentos.

Para obtener resultados de los niveles de compromiso y burnout, se trabajó con estadísticos descriptivos y luego se realizaron análisis de correlación de Pearson; para valorar el nivel predictivo de las variables se realizó la regresión lineal. Como prueba de comprobación de hipótesis se usó la prueba de chi cuadrado comparando la $\chi_{\text {prueba }}^{2} \operatorname{con} \chi_{\text {ta }}^{2}$. La fiabilidad de los instrumentos se realizó por medio del análisis de alfa de Cronbach.

\section{Resultados}

Los factores de la personalidad hallados en la muestra de docentes corresponden a responsabilidad, amabilidad, extroversión, neuroticismo y apertura en tres niveles de medición, como se puede apreciar en la Tabla 1. En la medición de los cinco grandes factores de la personalidad se encontraron rasgos que corresponden a características propias del individuo que interactúan con el ambiente en diferentes contextos de la vida. En este sentido, se estableció el rasgo compromiso al trabajo como uno de los principales asociados a la actividad docente, y se obtuvieron resultados asociados a niveles de compromiso alto en la muestra de análisis. De la misma manera, en el análisis de los resultados de los niveles de burnout se encontraron porcentajes significativos de entre el nivel medio y alto de agotamiento laboral (ver Tabla 2).

Tabla 1

Factores de personalidad.

\begin{tabular}{c|c|c|c|c|c|c} 
& \multicolumn{2}{|c|}{ Alto } & \multicolumn{2}{c|}{ Miveles } & \multicolumn{2}{c}{ Majo } \\
& f & $\mathbf{\%}$ & $\mathbf{f}$ & $\mathbf{\%}$ & f & \% \\
\hline Responsabilidad & 67 & 50 & 65 & 48.5 & 2 & 1.5 \\
Amabilidad & 30 & 22.4 & 73 & 54.5 & 31 & 23.1 \\
Extroversión & 38 & 28.4 & 73 & 54.5 & 23 & 17.2 \\
Neuroticismo & 49 & 36.6 & 58 & 43.3 & 27 & 20.1 \\
Apertura & 49 & 36.6 & 41 & 30.6 & 44 & 32.8
\end{tabular}

Fuente: Elaborada por los autores.

Tabla 2

Niveles de variables: Compromiso - Burnout.

\begin{tabular}{c|c|c|c|c}
\multirow{2}{*}{ Bajo } & \multicolumn{3}{|c|}{ Niveles de compromiso } & \multicolumn{3}{c}{ Niveles de burnout } \\
\cline { 2 - 5 } & $\mathbf{f}$ & $\mathbf{\%}$ & $\mathbf{f}$ & $\mathbf{\%}$ \\
\cline { 2 - 5 } & 8 & 6 & 1 & 0.7
\end{tabular}




\begin{tabular}{c|c|c|c|c} 
& \multicolumn{2}{|c|}{ Niveles de compromiso } & \multicolumn{2}{c}{ Niveles de burnout } \\
& f & \% & f & \% \\
\cline { 2 - 5 } Medio & 35 & 26.1 & 121 & 90.3 \\
Alto & 91 & 67.9 & 12 & 9 \\
Total & 134 & 100 & 134 & 100
\end{tabular}

Fuente: Elaboración de los autores

En la descripción de frecuencias de la medición de las dos variables por niveles se pudo observar la tendencia de los resultados en función al compromiso y al burnout. En este sentido y por medio de la correlación de Pearson, se analizó la relevancia de las variables entre ellas: (1) Compromiso - Burnout ( $r$ $=-.315 ; p=.001)$. En el análisis bilateral de la correlación negativa se puede interpretar que, entre menos niveles de compromiso, se pueden dar más altos niveles de burnout y asimismo, en dos factores de la personalidad: responsabilidad $(r=-.298 ; p=.000)$ y neuroticismo $(r=-.298 ; p=.001)$. Estos factores, si se presentan en niveles bajos, pueden aumentar los niveles de burnout.

Además, se analizaron indicadores de burnout asociados a los niveles de compro- miso, y se mostró la importancia de la integralidad de los procesos que se asocian al principal. En el estudio realizado, el compromiso fue señalado de importancia en el ámbito docente y abre campos de investigación para estudios sobre los rasgos y los factores de la personalidad como predictores de estabilidad emocional en el campo laboral (ver Tabla 3). Se evidenciaron correlaciones significativas negativas entre el elemento de agotamiento emocional y el factor de personalidad compromiso enfocado al trabajo (-.287) con una pendiente de regresión del $7.7 \%$. La despersonalización correlaciona significativamente y de manera negativa con el compromiso de apoyar (-.178) y el compromiso enfocado al trabajo (-.235) con una pendiente de regresión de $3.3 \%$ y de $4.98 \%$, respectivamente.

Tabla 3

Correlaciones entre variables.

\begin{tabular}{c|c|c|c|c|c|c|c|c|c} 
& \multicolumn{3}{|c|}{$\begin{array}{c}\text { Agotamiento } \\
\text { emocional }\end{array}$} & \multicolumn{2}{c|}{ Despersonalización } & \multicolumn{3}{|c|}{ Nivel burnout } \\
& $\boldsymbol{r}$ & $\boldsymbol{p}$ & $\boldsymbol{r}^{2}$ & $\boldsymbol{r}$ & $\boldsymbol{p}$ & $\boldsymbol{r}^{2}$ & $\boldsymbol{R}$ & $\boldsymbol{p}$ & $\boldsymbol{r}^{2}$ \\
$\begin{array}{c}\text { Compromiso, Inspira- } \\
\text { ción, Motivación }\end{array}$ & -.195 & .05 & & -.202 & .01 & & -.15 & .001 & .077 \\
Necesidad de apoyar & & & & -.178 & .04 & .033 & -2.35 & .006 & .049 \\
Enfocado al trabajo & -.172 & .04 & .02 & -.170 & .04 & .02 & -.278 & .001 & .077
\end{tabular}

Fuente: Elaborada por los autores

En el análisis de otros indicadores de las variables de estudio no se encontraron correlaciones entre la realización personal y el compromiso. Un aspecto importante de destacar es que el nivel de burnout correla- ciona negativamente con el compromiso tanto en el nivel de motivación (-.170), apoyo (-.170) y enfoque al trabajo (-.278), con el análisis de regresiones de $2.7 \%$ en los dos primeros casos y de $7.7 \%$ en el último. La antigüedad 
correlaciona con el compromiso en su nivel de motivación (-.172) con una pendiente de regresión de $2.7 \%$. Todos estos análisis se tomaron en cuenta con el nivel de significancia de $p<.05$. En el análisis de la prueba de hipótesis se comprobó la dependencia de los niveles de burnout con los niveles de compromiso $\left(\chi_{(4)}^{2}=11.9 ; p=.018\right)$. Siendo el valor estadístico $\left(\chi_{\text {prueba }}^{2}=11.9\right)$ mayor que el valor crítico de distribución de la tabla de chi cuadrado $\left(\chi_{\text {tabla }}^{2}=9.48\right)$, se acepta la hipótesis alternativa y se especifica la relación entre las variables compromiso-burnout. Además, se analiza el coeficiente de contingencia, usado para el análisis de relación entre variables cualitativas que obtiene un valor de .30 que, de acuerdo con la interpretación del grado del efecto según Cohen, existe un nivel medio de asociación entre las dos variables de análisis.

\section{Discusión y conclusiones}

Entre las conclusiones más importantes, se observa una conexión negativa entre el rasgo de personalidad compromiso y los niveles de burnout existente en las personas (-.315), así como con el agotamiento emocional (-.195) y la despersonalización (-.202), lo cual demuestra que, a medida que este rasgo es más débil, se fortalecen las dimensiones del agotamiento emocional y la despersonalización. Esto coincide con el aporte de Sánchez (2013) y con Pérez, Molero, Martos y Gásquez (2019), quienes hacen mención de una relación causal entre ambos.

El gran factor de la personalidad, responsabilidad, bajo el cual se encuentra el compromiso, valida la correlación negativa con el agotamiento emocional (-.199); la despersonalización (-.241) y de forma general con los niveles de burnout (-.298). Esto coincide con Glazer y Kruse (2008), quienes mencionan que el compromiso organizacional se puede convertir en un elemento protector ante situaciones estresantes, enunciado que también es apoyado por Dolatshah y Hosseini (2016).

En cuanto a los otros factores de persona- lidad, se puede mencionar que no se encontró relación entre los niveles de burnout y los grandes rasgos de personalidad de amabilidad y extraversión, pero sí se encontraron correlaciones entre el neuroticismo y los niveles de burnout y de despersonalización, lo cual difiere con los resultados de Laca, Mejía, Yañez y Mayoral (2011), quienes encontraron que el rasgo amabilidad actúa como un predictor de la realización personal.

La dimensión de realización personal no correlaciona con ninguno de los cinco grandes factores de personalidad y tampoco lo hace con el factor compromiso y las escalas que lo conforman, lo cual difiere de los resultados de Gordon (2004), quien mencionaba un vínculo entre la realización personal y el compromiso. Tampoco se encontró en este estudio una relación entre la antigüedad de los docentes y los niveles bajos de realización personal, como lo menciona Sagripanti, González, Messi, Romero y Khlie (2012).

En referencia a la clasificación diagnóstica que posee el Manual de Diagnóstico Estadístico (DSM IV) y la Clasificación Internacional de Enfermedades (CIE 10) acerca del Síndrome de Burnout, se puede observar que en los manuales diagnósticos no se establece categorización de la entidad per se. Más aún, existen tipos de trastornos que se asemejan en su sintomatología; por ejemplo, si hay un trabajador afectado de burnout y su adaptabilidad a un sistema laboral no es adecuado, se puede, en este caso, por principio de semejanza, dar una categorización en referencia a los trastornos de tipo adaptativo, los cuales refieren en sus criterios síntomas emocionales o comportamentales en respuesta a un estresante identificable.

En el análisis de la prueba de hipótesis se comprobó la dependencia de los niveles de burnout con los niveles de compromiso, lo cual coincide con Ortega y Ortiz (2014), quienes consideran a la personalidad como un moderador de los niveles de burnout.

Las implicaciones del estudio pueden ser de utilidad para mejorar la calidad del sistema 
educativo, en el que el rol principal lo tiene el profesorado. Por este motivo, estos resultados podrían ser orientadores para incorporar nuevas formas de trabajo del docente e implementar mejoras en procesos de selección, en el que el perfil exija niveles altos de responsabilidad en su rasgo de personalidad para, de esta manera, mejorar su bienestar e incrementar la productividad de las organizaciones educativas. Esto contribuiría a mejorar la calidad de vida los docentes.

En estudios próximos se sugiere indagar en otros factores de la personalidad que están ligados al gran factor responsabilidad, como son los roles de planificar, mantener el orden y las necesidades de terminar una tarea o conseguir las metas. El tipo de muestra se convirtió en una limitación, por lo que se considera conveniente para próximos estudios trazar una ruta aleatoria que abarque una zona más amplia, la cual permita realizar una comparación entre unidades educativas que posean bachillerato internacional.

\section{Referencias bibliográficas}

Aldrete, M. G., Navarro, C., González, R., Contreras, M. y Pérez, J. (2015). Factores Psicosociales y Síndrome de Burnout en Personal de Enfermería de una unidad de Tercer Nivel de Atención a la Salud. Ciencia y Trabajo, 17(52), 32-36. https://doi.org/10.4067/S071824492015000100007

Arenas, F. y Andrade, V. (2013). Factores de Riesgo Psicosocial y Compromiso con el trabajo en una organización del sector salud de la Ciudad de Cali, Colombia. Acta Colombiana de Psicología, 16(1), 43-56.

Arias Gallegos, W. L. y Núñez Cohello, A. L. (2015). Síndrome de Burnout en Supervisores de Seguridad Industrial de Arequipa. Ciencia y Trabajo, 17(52), 77-82. https://doi. org/10.4067/S0718-24492015000100013

Arquero, J. L., Donoso, J. A., Hassall, T. y Joyce, J. (2006). El Impacto del Síndrome del Burnout en los Profesores Noveles. Un estudio piloto. Revista de Enseñanza Universitaria,(27),
69-82.

Association American Psychiatric. (1995). Manual diagnóstico y estadístico de los trastornos mentales. Barcelona: Masson.

Böhrt, R., Solares, L. y Romero, C. (2014). Evolución del contrato psicológico y el compromiso organizacional con la edad y la antiguedad. Ajayu, 12(1), 33-63.

Casademunt, A. L., Morales-Gutiérrez, A. C. y Ariza-Montes, J. A. (2012). La implicación emocional en el puesto de trabajo: Un estudio empírico. Intangible Capital, 8(2), 364-405. https://doi.org/10.3926/ic.321

Castro, D., Martínez, F., Robledo, D. y Sierra, E. (2014). Compromiso Organizacional en trabajadores del rubro minero de la IV Región. Revista de Psicología - Univerisidad Mar del Plata, 3(6), 34-51.

Contreras, F., Espinal, L., Panchón, A. M. y González, J. (2013). Burnout, liderazgo y satisfacción laboral en el personal asistencial de un hospital de tercer nivel en Bogotá. Diversitas: Perspectivas en Psicología, 9(1), 65-80. https:// doi.org/10.15332/s1794-9998.2013.0001.05

Cornejo Chávez, R. (2009). Condiciones de trabajo y bienestar/malestar Docente en profesores de enseñanza media de Santiago de Chile. Educación y Sociedad, 30(107), 409-426.

Cubiks. (2014). Manual del Usuario. Cubiks Intellectual Property Limited.

Dolatshah, A. y Hosseini, M. (2016). The Relationship between Cultural Intelligence and Organizational Commitment and Burnout among Employees of the Arman Financial Institution. International Journal of Management Science, 6(12), 595-603.

Gago, K., Martínez, I.y Alegre, A. (2017). Clima laboral y síndrome de burnout en enfermeras de un hospital general de Huancayo, Perú. Psiencia Revista Latinoamerica de Ciencia Psicológica, 9, 1-15. https://doi.org/10.5872/ psiencia/9.4.21

García Izquierdo, A. L., Ramos Villagrasa, P. J.y García Izquierdo, M. (2009). Los Big Five y el Efecto moderador de la Resistencia en el Agotamiento Emocional. Revista de Psicología del Trabajo y de las organizaciones, 135-147. 
Garrosa, E., Rainho, C., Moreno, B. y Monteiro, M. (2010). The relationship between job stressors, hardy personality, coping resources and burnout in a sample of nurses: A correlational study at two time points. International Journal of Nursing Studies, 47, 205-215. https://doi.org/10.1016/j.ijnurstu.2009.05.014

Gholipour, A., Reza, M., Anvari, A. y Kalali, N. S. (2011). Investigation of the Effects of the Big Five Personality Model on Job Burnout (Survey in an Iranian Hospital). International Proceedings of Economics Development and Research, 4, 171-178. https://

pdfs.semanticscholar.org/cdcf/5bf61940e2958b9ad3a7844aa6a549328b4b.pdf

Giménez, S., Gilla, M., Morán, V. y Olaz, F. (2019). Burnout y sintomatología psicopatológica en profesionales argentinos de la salud mental. Psiencia Revista Latinoamericana de Ciencia Psicológica, 10, 1-20. https://doi. org/10.5872/psiencia/10.3.22

Glazer, S. y Kruse, B. (2008). The Role of Organizational Commitment in Occupational Stress Models. international journal of stress management, 329-344. https://psycnet.apa.org/ doi/10.1037/a0013135

Gordon, H. (2004). The relationship between burnout and organizational commitment in academic oncologist. Dissertation Abstracts International: Section B: The sciences and Engineering, 64(8-B), 3730.

Hederich, C. y Caballero, C. (2016). Validación del Cuestionario Maslach Inventory- Student Survey (MBI-SS) en contexto académico colombiano. Revista CES Psicología, 1-15.

Heinemann, L. V. y Heinemann, T. (2017). Burnout Research: Emergence and Scientific Investigation of a Contested Diagnosis. SAGE Open, 1-12. https://doi. org/10.1177\%2F2158244017697154

Jeong Kim, H., Hyun Shin, K. y Swanger, N. (2009). Burnout and engagement: A comparative analysis using the Big Five personality dimensions. International Journal of Hospitality Management, 28, 96-104. https://doi. org/10.1016/j.ijhm.2008.06.001

Kokkinos, C. (2007). Job stressors, person- ality and burnout in primary school teachers. British Journal of Educational Psychology, 77, 229-243. https://doi. org/10.1348/000709905X90344

Laca Arocena, F. A., Mejía Ceballos, J. C., Yáñez Velasco, C. L. y Mayoral Sánchez, E. G. (2011). Factores afectivos y de personalidad como antecedentes del bienestar laboral en profesores españoles y mexicanos. Pensamiento Psicológico, 9(16), 11-26.

Lewis, C. y Anderson, P. (1998). PAPI technical manual. Cubiks.

Llanque, P. (2014). Rasgos de Personalidad y Síndrome de Burnout en Personal de Salud de la Unidad de Quemados y Cirugía Plástica del "Hospital de Clínicas" de la Ciudad de La Paz. Lecturas en Psicología, 93-115.

Lupano, M. y Castro, A. (2018). Influencia de virtudes organizacionales sobre satisfacción, compromiso y performance laboral en organizaciones argentinas. Interdisciplinaria, 35, 171-188. https://doi.org/10.16888/ interd.2018.35.1.9

Malander, N. M. (2016). Síndrome de burnout y satisfacción laboral en docentes de nivel secundario. Ciencia \& Trabajo, 177-182. doi:https:// doi.org/10.4067/S0718-24492016000300177

Maldonado-Radillo, S. E., Ramírez Barón, M. C., García Rivera, B. R. y Chairez Venegas, A. (2014). Compromiso Organizacional de los Profesores de una Universidad Pública. Conciencia Tecnológica, (47), 12-18.

Marenco-Escuderos, A. D. y Ávila-Toscano, J. H. (2016). Burnout y problemas de salud mental en docentes: diferencias según características demográficas y sociolaborales. Psychologia: Avances de la Disciplina, (10), 91-100

Marsollier, R. (2013). La despersonalización y su incidencia en los procesos de desgaste laboral. Psicologia.com

Martínez Ramón, J. P. (2015). Como se defiende el profesorado de secundaria del estrés: burnout y estrategias de afrontamiento. Revista de Psicología del Trabajo y de las Organizaciones, 31(1), 1-9. https://doi.org/10.1016/j. rpto.2015.02.001

Maslach, C. y Jackson, S. (1986). Maslach 
Burnout Inventory. Palo Alto: Pysichologists Press.

McCrae, R. R. y Terracciano, A. (2005). Personality Profiles of Cultures: Aggregate Personaity Traits. Journal of Personality and Social Psycoogy, 89(3), 407-425. https://doi. org/10.1037/0022-3514.89.3.407

McCrae, R. R., Costa Jr, P. T., del Pilar, G. H., Rolland, J. P. y Parker, W. D. (1998). Cross-cultural assessment of the five-factor model: The Revised NEO Personality Inventory. Journal of Cross- Cultural Psychology, 29, 171-188. https://doi.org/10.1177/0022022198291009

Mejía, J. C., Laca, F. A. y Gondra, J. M. (2009). Factores de personalidad, afectivos y sociodemográficos en la predicción del bienestar laboral de docentes. Psicología y Salud, 19(1), 121-132.https://doi.org/10.25009/pys. v19i1.652

Mischel, W. (2004). Towards an integrative science of the person. Annual Review of Psychology, 55, 1-22. https://doi.org/10.1146/ annurev.psych.55.042902.130709

Morán Astorga, C. (2005). Personalidad, Afrontamiento y burnout en profesionales de atención a personas con discapacidad intelectual. Siglo Cero: Revista Española sobre discapacidad intelectual, 30-39.

Moreno, B., González, J. y Garrosa, E. (2001). Desgaste profesional (burnout), Personalidad y Salud percibida. Empleo, estrés y salud, 59-83. Narumoto, J., Nakaruma, K., Kitabayashi, Y., Shibata, K., Nakamae, T. y Fukui, K. (2008). Relationships among burnout, coping style and personality:Study of Japanese professional caregivers for elderly. Psychiatry and Clinical Neurosciences, 62, 174-176.https://doi. org/10.1111/j.1440-1819.2008.01751.x.

Navarro Abal, Y. (2009). ¿Influyen los rasgos de personalidad y el método docente empleado en la percepción del rendimiento académico del alumnado universitario? Revista Qurriculum, 189-206.

Ortega, M. E. y Ortiz, G. R. (2014). Burnout en estudiantes de pregrado de medicina $\mathrm{y}$ su relación con variables de personalidad. Terapia Psicológica, 32, 235-242. https://doi.
org/10.4067/S0718-48082014000300006

Pando, M., Gascón, S., Varillas, W. y Aranda, C. (2019). Exposición a factores psicosociales laborales y sintomatología de estrés en trabajadores peruanos. Ciencia Unemi, 12, 01 - 08. https://doi.org/10.29076/issn.2528-7737vo112iss29.2019pp1-8p

Perez, M., Molero, M., Martos, A. y Gasquez, J. (2019). Burnout and Engagement: Personality Profiles in Nursing Professionals. Clinical Medicine, 1-14.

Popp, M. (2008). Estudio preliminar sobre el síndrome de Burnout y estrategias de afrontamiento en enfermeras de unidades de terapia intensiva (UTI). Inderdisciplinaria, 25, 5-27.

Prada-Ospina, R. (2019). Relación de factores sociales y psicológicos con el estrés laboral y su vínculo con el síndrome de burnout. Interdisciplinaria, 36, 39-53.

Ramírez, M. y Lee, S. L. (2012). Síndrome de burnout entre hombres y mujeres medido por el clima y la satisfacción laboral. Polis, 1-15.

Rodríguez, R. y De Rivas, S. (2011). Los procesos de estrés laboral y desgaste profesional (burnout): diferenciación, actualización y líneas de intervención. Medicina y Seguridad del Trabajo, 72-88.

Ruíz, D., Pando, M., Aranda, C. y Almeida, C. (2014). Burnout y Work Engagement en Docentes Universitarios de Zacatecas. Ciencia $y$ Trabajo, 16(50), 116-120.

Sagripanti, O. G., Gonzalez, M. J., Messi, I., Romero, J. y Khlie, Y. (2012). El efecto de burnout en la antiguedad en el puesto de trabajo: Análisis mediacional de la ansiedad. Revista electrónica de investigación Docencia Creativa, 1, 4-11.

Sánchez, D., Sánchez, P., Pulido, J., López, J. M. y Cuevas, R. (2014). Motivavión y burnout en profesores de educación física: incidencia de la frustración de las necesidades psicológicas básicas. Cuadernos de psicología del deporte, 14, 75-82.

Sánchez, M. I. (2013). Compromiso Laboral y Estrés en los empleados de Bancos y Cajas. Pecvenia (16-17), 85-100.

Sanz, J., Gil, F., Barrasa, A. y García, M. (2006). 
Self-assessment of needs and behavior patterns at work:Psychometric properties of the Personality and Preference Inventory-Normative (PAPI-N). Personality and Individual Differences, 41(5), 837-847. https://doi. org/10.1016/j.paid.2006.03.019

Silva Henao, D. C., Gutiérrez Strauss, A. M., Pando Moreno, M. y Tuesca Molina, R. (2014). Relación entre factores psicosociales negativos y el síndrome de Burnout en el personal sanitario de Florencia (Caquetá, Colombia). Salud Uninorte, 30(1), 52-62.

Simkin , H. y Pérez Marín, M. (2018). Personalidad y Autoestima: Un análisis sobre el importante papel de sus relaciones. Terapia Psicológica, 19-25.

Spontón, C., Trógolo, M., Castellano, E. y Medrano, L. (2019). Medición del burnout: Estructura factorial, validez y confiabilidad en trabajadores argentinos. Interdisciplinaria, 36(1), 87-103. https://doi.org/10.16888/ interd.2019.36.1.7
Suárez, Y. P. y Mendoza, J. A. (2014). Indicadores de salud mental y engagement en empleados de una empresa promotora del desarrollo económico. Económicas CUC, 35(2), 133-146.

Vallejo Sánchez, B. y Pérez García, A. (2018). Contribución del Neuroticismo, rasgos patoógicos de persobalidad y afrontamiento en la predicción de la evolución clínica: estudio de seguimiento da los 5 años de una muestra de pacientes con trastorno adaptativo. Clínica $y$ Salud, 58-62.

Vásquez-Manrique, J. F., Maruy-Saito, A. y VerneMartin, E. (2014). Frecuencia del síndrome de Burnout y niveles de sus dimensiones en el personal de salud del servicio de emergencia de pediatría del Hospital Nacional Cayetano Heredia en el año 2014. Lima, Perú. Revista de Neuro-Psiquiatría, 77(3), 168-174.

World Health Organization. (2000). Guia de bolsillo de la clasificación CIE-10 : clasificación de los trastornos mentales y del comportamiento. Médica Panamericana.

Recibido: 25 de octubre de 2019

Aceptado: 23 de febrero de 2021 